\title{
A Two-Phased PSO-SVM Predictive Quality Control Model for Multistage Production
}

\author{
Yang Jingping \\ College of Electromechanical \& Information \\ Engineering, \\ Dalian University of Nationalities \\ Dalian, China \\ e-mail: yjp@dlnu.edu.cn \\ Wang Wanlei \\ College of Electromechanical \& Information \\ Engineering, \\ Dalian University of Nationalities \\ Dalian, China \\ e-mail: wwl@dlnu.edu.cn
}

\author{
Luo Yuegang \\ College of Electromechanical \& Information \\ Engineering \\ Dalian University of Nationalities \\ Dalian, China \\ e-mail: luoyg@dlnu.edu.cn \\ Kang Jing \\ College of Electromechanical \& Information \\ Engineering, \\ Dalian University of Nationalities \\ Dalian, China \\ e-mail: kj@dlnu.edu.cn
}

\begin{abstract}
Steel production is regarded as a typical multistage system given its long process flow feature. In this paper, the quality predictive control features of steel production is studied; a two-phased PSO-SVM predictive control model for multi-stage production is established. In order to solve the model, a constrained PSO hyper kernel parameters optimization algorithm with a PCA data pre-processing method is proposed. The model is tested and proved to be effective by quality data of a steel production enterprise, in the case, the prediction of the multi-stage production is realized in phase one, and the global optimization of related process parameters is worked out in phase two.
\end{abstract}

Keywords-quality predictive control; process parameters optimization; support vector machine (SVM); hierarchical model; particle swarm optimization (PSO)

\section{INTRODUCTION}

Although the most reliable approach to quality improvement will be the use of precise first-principle models, such models are not available in most newly developed processes and modeling of a complex industrial process is very difficult and time-consuming ${ }^{11}$. Meanwhile, with applications of information management systems such as MES, enterprises have stored lots of original data relative to production and quality. These data can be a good reflection to enterprise's production conditions and product quality if we can take good advantage of them. In recent years, a research trend is forming to analyze the relationships between the product quality and relative process parameters on the basis of on these data to support production decision makings and quality assurance, and model predictive control technologies based on Data Mining and other intelligent method is a new way to solve this kind of problem ${ }^{[2]}$. Take the typical multi-stage complex steel production process for an example, there exist a number of research results, such as a hybrid model with the combination of a predictive artificial neural network (ANN) and a genetic algorithm (GA) ${ }^{[3]}$, a BP neural network multivariate time series model and T-S fuzzy neural network model of multivariate time series for blast furnace temperature control ${ }^{[4]}$, etc. These researches focus on the prediction and control of single quality indices, and there are some new researches on multistage or multiphase processes ${ }^{[5,6]}$, too. In this paper, we will try to solve this kind of problem by a new hierarchical SVM predictive quality control model.

\section{SYSTEM DESCRIPTIONS}

\section{A. Quality control features of multistage production}

Multistage production such as iron and steel production has a relatively long, continuous and complicated process. Typical iron and steel production is composite of three main stages: steel making, billet rolling and product rolling. Although within each stage, processes are continuous, between stages, they are more likely to be managed and controlled separately.

Yield of the product which should be calculated after key processes can directly reflect the qualified output rate, so it is a commonly monitored synthetic quality index for steel and iron enterprises. Due to the different factors in each stage such as equipments, processes, etc., the yield expression of each stage is different. The final yield of the product can be obtained by the product of all the stages, as shown in equation (1), where $y_{s}$ stand for the final yield of the product, and $y_{i}$ stand for yield of the stage $i$.

$$
y_{\mathrm{s}}=\prod_{i=1}^{n} y_{i}
$$

\section{B. Quality control model and parameters}

In the Iron and steel production processes, the factors affecting the yield of different stages are various. In the steel making process, the factors mainly include: the purity and the ratio of raw materials, auxiliary materials (such as purity of oxidizer, pressure and moisture, the components, size of other materials, etc.), smelting 
process parameters (such as the mixture, tapping temperature, killed steel time, tapping time, casting time etc.). In the billet rolling process, main factors include: the chemical ingredients, ingot surface defects, ingot quality problems (such as the impurities), ingot type, ingot heating speed, heating time, heating evenness, rolling speed, cooling time, grinding or peeling quantities etc. In the product rolling process, main factors include: billet surface quality, the internal quality of billet, rolling process parameters, etc. Due to the development of steelmaking industrial, rolling metallurgical professional technology theory, some relationship between factors and the quality of steel products have been mastered by the engineers, but because of the complexity of the actual production process environment, and the high dimension, nonlinear and strong correlation features of the factors, the problems become particularly complex.

The core thought of the predictive control of yield is to improve yield rate by researching the relationship between yield and relative process parameters according to historical data, predicting the probable value and the changing trend of yield under various process parameters, and optimizing process parameters to improve process quality. The process parameters related to yield of stages are scared in processes, and with non-unified forms. They should be analyzed, transformed and homogenized to provide complete process sample information for the prediction of yield. The universal set of the process parameters can be noted as $P=\left\{p_{1}, p_{2}, \mathrm{~L}, p_{m}\right\}$, and the matrix $C_{n \times m}=\left[\begin{array}{ccc}c_{11} & \mathrm{~L} & c_{1 m} \\ \mathrm{M} & \mathrm{O} & \mathrm{M} \\ c_{n 1} & \mathrm{~L} & c_{n m}\end{array}\right]$ is constructed to set the relationship between the yield of each stages and each process parameter, when yield $y_{i}$ and process parameter $p_{j}$ have relationships, the corresponding $c_{i j}$ would be set as 1 , and otherwise, 0 would be set to the corresponding $c_{i j}$.

Since the factors related to the yield rate of different stages are not always the same, if we set up a single regression model for the whole production, not only a number of inputs and outputs would lead to a large dimension model, but also a variety of interacted parameters would cause a inaccurate prediction result. Instead of that, we make relatively special models for each stage according to each own factors, respectively. By this means, not only synchronization analysis of each stage can be realized, but also the abnormal process status of each stage can be efficiently and accurately monitored.

For each stage, the decision function of yield can be noted as $y_{1}, \mathrm{~L}, y_{i}, \mathrm{~L}, y_{n}$. So, the quality control model can be expressed as the series connection of the decision functions, as shown in equation (2).

$$
\begin{gathered}
y_{s}=\prod_{i=1}^{n} y_{i}=\prod_{i=1}^{n} f\left(c_{11} p_{1}, \mathrm{~L}, c_{i j} p_{j}, \mathrm{~L}, c_{i m} p_{m}\right) \\
\text { Where: } \quad c_{i j}=\left\{\begin{array}{l}
0, \text { where } p_{j} \text { isn't related to } y_{i} \\
1, \text { where } p_{j} \text { is related to } y_{i}
\end{array}\right.
\end{gathered}
$$

\section{PSO-SVM BASED PRODUCT QUALITY PREDICTIVE MODEL}

\section{A. Introduction to related theories}

Due to the ability to learn well with only a very small number of free parameters, the robustness against several types of model violations and outliers, and the computational efficiency compared with several other methods, support vector machine is a successful modeling and prediction tool based on statistical learning and structural risk minimization principle which firstly proposed by Vapnik in $1995^{[7]}$.

In recent years, based on the standard SVM, many extended studies and application of SVM are carried out, such as the asymmetric least squares SVM classifiers ${ }^{[8]}$. In order to reduce the complexity of prediction control model, PCA, Kernel PCA (KPCA) and Independent Component Analysis (ICA) are applied for data compression when using support vector machine ${ }^{[9]}$.

\section{B. PSO-SVM prediction control process model}

Because of the complexity, time-varying and nonlinear characteristics of the multistage production, it is very hard to build an accurate model. Although classical process modeling method such as PCA (principal component analysis) and PLS (partial least square) methods can be used to establish a quality regression model, they cannot handle the nonlinear dynamical problems. In this paper, take steel and iron industry for an example, based on the principal of SVM (supported vector machine), a PSOSVM predictive control process model for multistage synthetic yield control as shown in Fig .1 is constructed, where rough set theory and PCA is applied as a data preprocessing and compression method, and an improved particle swarm algorithm is introduced to solve the model.

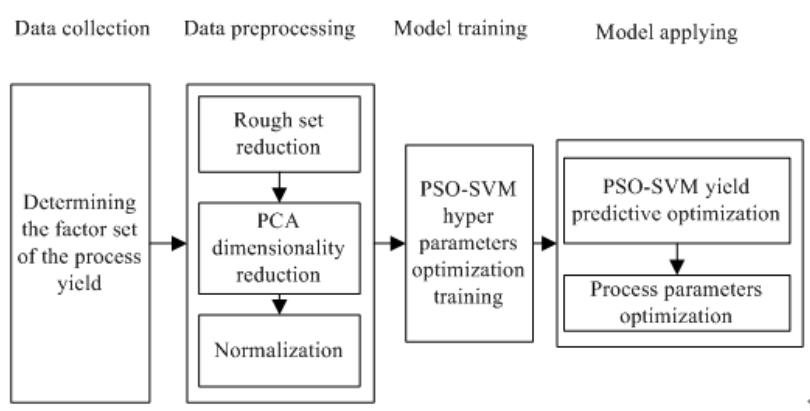

Figure 1. PSO-SVM prediction control process model.

- $\quad$ A RS-PCA based data pre-processing method

Data mining (also known as knowledge discovery in databases) is the process of extracting patterns from large data sets to analyze data from different perspectives and summarize them into useful information ${ }^{[10]}$. Rough Set (RS) theory proposed by Z. Pawlak is an excellent mathematical tool to deal with inconsistency and ambiguity information for data mining; it focuses on the approximation of a concept by using blocks induced by a binary relation. Relational tables of Data mining can be used as information tables of rough set theory, by this means, reduction theory of rough set can be used for the preprocess of high dimensional data to remove redundant attributes, so as to reduce the dimension of the data. 
Applying RS theory can efficiently reduce the dimension of the factors, but still, it can't deal with correlated factors. In order to solve this problem, principal component analysis (PCA) is utilized on the original data by which in one hand, the error caused by modeling on the correlated input variables of parallel regression functions can be eliminated, in another hand, the model complexity can be reduced under the guarantee of the model accuracy.

- $\quad$ PSO-SVM prediction model and the construction of kernel function

The aim of the quality control model is to find a suitable process parameters set $X=\left\{x_{1}, x_{2}, \mathrm{~L}, x_{m}\right\}$ of an equivalence relation set $R_{B}$ worked out according to RS theory, where

$R_{B}=\left\{\left(p_{j} c_{i j}, x_{j}\right) \in U \times U \mid f\left(p_{j} c_{i j}, a\right)=f\left(x_{j}, a\right)\right\}$.

So the corresponding SVM model proposed in this paper is:

$$
y=\prod_{k=1}^{n} \sum_{i=1}^{s}\left(\left(\bar{\alpha}_{i}^{*}-\alpha_{i}^{*}\right) K(X, \quad X)+\not\right)
$$

Where $X^{\prime}=A^{T} X$ is the PCA vector, and $b_{1}, b_{2}, \mathrm{~L} b_{k}$ are the biases.

During the application of SVM, it is vital to select an appropriate kernel function, but there are no clear directions of how to choose kernel functions by now. Commonly used kernels include:

1) Linear kernel function: $K\left(x, x_{i}\right)=\left(x, x_{i}\right)$

2) Poly kernel function:

$$
K\left(x, x_{i}\right)=\left[\left(x, x_{i}\right)+1\right]^{q}, q \in N
$$

3) Radial basic function:

$$
K\left(x, x_{i}\right)=\exp \left(-\gamma\left\|x, x_{i}\right\|^{2}\right), \gamma>0
$$

4) Sigmoid kernel function:

$$
K\left(x, x_{i}\right)=\tanh \left(v\left(x, x_{i}\right)+c\right)
$$

Where RBF is a typical local kernel function with strong study abilities, and Poly is a global kernel function with high generalization abilities. Hence, in this paper, a mixed kernel as equation (4) is used.

$$
K\left(x, x_{i}\right)=\omega K_{\text {poly }}+\left(1-\omega K_{R B F}\right), 0 \leq \lambda \leq 1
$$

\section{TWO PHASED PSO PREDICTION ALGORITHM BASED ON MULTI-CONSTRAINED PSO COMBINED WITH SVM}

Particle swarm optimization (PSO) is an evolutionary algorithm known for its simplicity and effectiveness in solving various optimization problems, while SVM has a strong learning ability with small samples, so, in recent studies, the PSO algorithm is widely used to solve the hyper parameters selection of support vector machine ${ }^{[11]}$. In this paper, a constrained PSO-SVM regression method is proposed to adjust and select both hyper parameters of SVM and process factors which lead to an optimized yield in the process quality prediction control process of the steel and iron manufacturing.

In the constrained particle swarm algorithm proposed in this paper, we let each particle represents a feasible solution. At the model training and prediction phase, the particles are encoded as the format of hyper parameters of the support vector machine model for each control point, i.e.

$$
Z_{1}=\left[k_{1}^{(1)}, \mathrm{L}, k_{s}^{(1)}, \mathrm{L}, k_{1}^{(i)}, \mathrm{L} k_{l}^{(i)}, \mathrm{L}, k_{s}^{(i)}, \mathrm{L}, k_{1}^{(m)}, \mathrm{L}, k_{s}^{(m)}\right] \text {, }
$$

where $k_{l}^{(i)}$ represents hyper parameters $l$ for control points $i, s$ represents the quantity of the main hyper parameters of each control points. Because of using the same kernel in each stage, so the main core of each stage is the same in quantity; $m$ is the total quantity of the control points. While at optimization of the process conditions stage, the particles are encoded format as the format of process parameters of each control point, i.e.

$$
Z_{2}=\left[\mathrm{L}, p_{j_{1}}^{(1)}, \mathrm{L}, p_{j_{i}}^{(i)}, \mathrm{L}, p_{j_{m}}^{(m)}, \mathrm{L}\right] \subseteq P,
$$

Where $p_{j_{i}}^{(i)}$ represents process condition $j_{i}$ for control point $i$, and the collection of the process conditions of each control point is included in the total conditions set $P$.

Since the PSO is not sensitive to the initial solution, according to the equation (5), random variable value of any sampling within their space constraints can be taken as an initial position:

$$
x_{u}^{(i)}=r_{x}\left(x_{u \max }^{(i)}-x_{u \min }^{(i)}\right)+x_{u \min }^{(i)}
$$

Where $r_{x}$ is a random number between $[0,1]$, $x_{u \max }^{(i)}$ and $x_{u \min }^{(i)}$ is maximum and minimum value of sample $i$.

The initial velocity of samples can be expressed as follows:

$$
v_{u}^{(i)}(0)=r_{v} v_{u \max }^{(i)}
$$

Where $r_{v}$ is a random number between $[0,1], \quad v_{u \max }^{(i)}$ is the maximum speed of sample $i$.

The optimum result can be worked out by PSO algorithm through tracing the best position. On each iteration, each $z_{u}$ can be solved with equation (7) and (8) :

$$
\begin{gathered}
z_{u}{ }^{(i)}(t+1)=z_{u}{ }^{(i)}(t)+v_{u}{ }^{(i)}(t+1) \\
v_{u}{ }^{(i)}(t+1)=w v_{u}{ }^{(i)}(t)+c_{1} r_{1}\left(p_{u}{ }^{(i)}(t)-x_{u}{ }^{(i)}(t)\right) \\
+c_{2} r_{2}\left(p_{u}{ }^{(i)}(t)-x_{u}{ }^{(i)}(t)\right)
\end{gathered}
$$

Where $t$ is the iteration times, $w$ is the inertia weight, $c_{1}$ and $c_{2}$ are acceleration constants, which are generally valued between $[0,2]$.

The adaptability of the particle swarms are verified according to the result of the prediction model, the objective function is as follows:

$$
f_{\max }=\prod_{k=1}^{m} y(k)=\prod_{k=1}^{n} \sum_{i=1}^{s}\left(\left(\bar{\alpha}_{i}^{*}-\alpha_{i}^{*}\right) K\left(X^{\prime}, X_{i}\right)+b_{k}\right)
$$

The best position particles experienced can be worked out by equation (10):

$$
p(t+1)=\left\{\begin{array}{l}
p(t), f(p(t+1)) \geq f(p(t)) \\
p(t+1), f(p(t+1))<f(p(t))
\end{array}\right.
$$

The two-phased PSO prediction algorithm process is shown as Fig. 2 . 


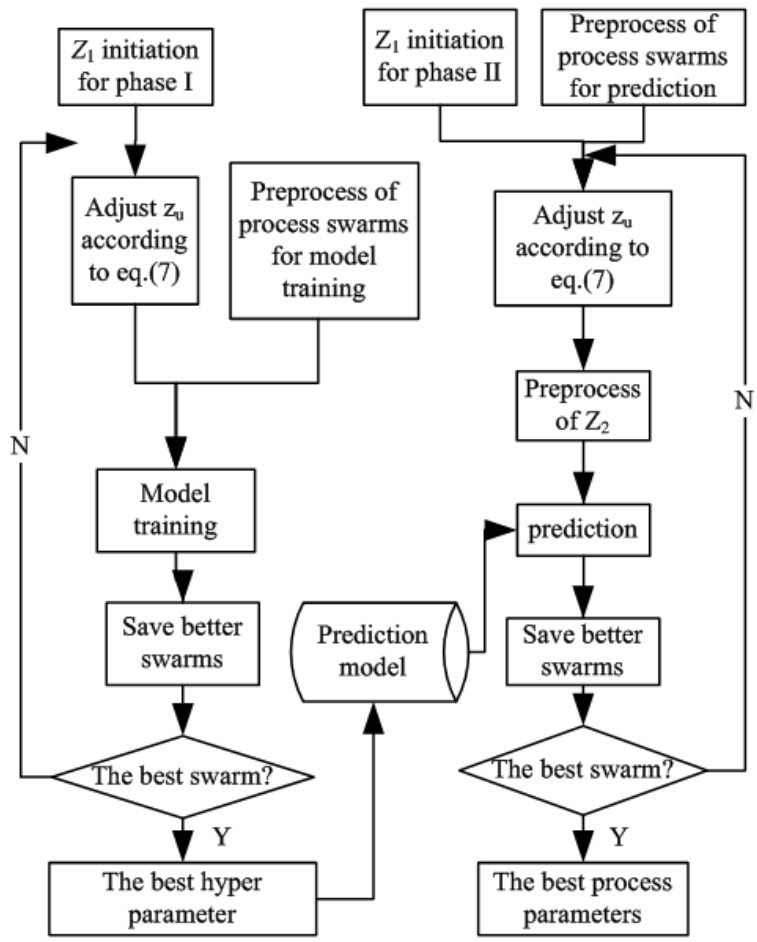

Figure 2. Two-phased PSO prediction algorithm process.

\section{CASE STUdy}

In this case, we take the typical process of a stainless steel wire as an example, which has processes include: smelting (0.994), blooming (0.85), and rolling (0.925), numbers in brackets are the planed yield of each process stage, so the total planed yield would be $0.994 * 0.85 * 0.925=0.78$.

Firstly, possible related factors (such as main materials, auxiliary materials, process parameters, chemical compositions) are collected and reduced by corresponding RS combined PCA functions realized by Excel functions. Then the hyper parameters of SVM are optimized by using the PSO algorithm programmed in Matlab, with an error penalty coefficient $C=100$, and a maximum allowed regression error $\varepsilon=0.005$, particles are set as the hyper parameters of the prediction model for each control point $\begin{aligned} & {\left[\lambda_{1}, q_{1}, \gamma_{1}, \lambda_{2}, q_{2}, \gamma_{2}, \lambda_{3}, q_{3}, \gamma_{3}\right] } \\ & =[1,9,0.9,0.485,3,0.024,1,8,0.9]\end{aligned}$, part of the optimization results of phase one are shown in TABLE 1.

TABLE I. TRAINING RESULT OF THE PREDICTION MODEL

\begin{tabular}{|c|c|c|c|c|c|}
\hline \multicolumn{2}{|c|}{ sample } & 1 & 2 & $\cdots$ & RMSE \\
\hline \multirow{3}{*}{$\begin{array}{c}\text { Stage } \\
1\end{array}$} & actual value & 0.993 & 0.975 & $\cdots$ & \multirow{3}{*}{0.040} \\
\hline & predict value & 0.973 & 0.972 & $\cdots$ & \\
\hline & error & -0.020 & -0.003 & $\cdots$ & \\
\hline \multirow{3}{*}{$\begin{array}{c}\text { Stage } \\
2\end{array}$} & actual value & 0.880 & 0.900 & $\cdots$ & \multirow{3}{*}{0.020} \\
\hline & predict value & 0.877 & 0.899 & $\cdots$ & \\
\hline & error & -0.003 & -0.001 & $\cdots$ & \\
\hline \multirow{3}{*}{$\begin{array}{c}\text { Stage } \\
\mathbf{3}\end{array}$} & actual value & 0.930 & 0.940 & $\cdots$ & \multirow{3}{*}{0.043} \\
\hline & predict value & 0.917 & 0.903 & $\cdots$ & \\
\hline & error & -0.013 & -0.037 & $\cdots$ & \\
\hline
\end{tabular}

At phase two, the process parameters of the maximum yield rate of the trained model are optimized by using particle swarm optimization algorithm, taking all the 28 process parameters into the particle set $\mathrm{S}$, including 11 elements of stage 1,15 elements of stage 2, and 17 elements of stage 3 , some elements belong to more than one stage. Set error penalty coefficient $C=100$, the maximum error allowed of regression $\varepsilon=0.005$, PSO iteration number is 1000 , the optimization results are obtained, and the final optimized integrated yield is: $0.963 * 0.925 * 0.916 \approx 0.816$.

\section{CONCLUSIONS}

The results we obtained demonstrate that the prediction quality control model proposed in this paper can take a fast and efficient approximation to historical data of quality characteristics. In this case, the predictive control optimization quality yield value $(0.816)$ was increased by $4.6 \%$ than the planned yield value (0.78). More importantly, the RS theory and PCA method are involved in the model to reduce quantities of related parameters, which makes the model and its algorithm simple to apply.

\section{ACKNOWLEDGMENT}

This paper is supported by "the Fundamental Research Funds for the Central Universities".

\section{REFERENCES}

[1] Manabu Kano, Yoshiaki Nakagawa, "Data-based process monitoring, process control, and quality improvement: Recent developments and applications in steel industry," Computers and Chemical Engineering, vol. 32, pp. 12-24, 2008

[2] Eduardo F. Camacho, Carlos Bordons, "Nonlinear Model Predictive Control: An Introductory Review,” in Springer, 2007.

[3] Shylu John, Sudipta Sikdar, P. Kumar Swamy, Sumitesh Das, Bhagabat Maity, "Hybrid neural-GA model to predict and minimise flatness value of hot rolled strips," Journal of Materials Processing Technology, vol.195, pp.314-320, January 2008.

[4] CUI Gui-mei, LI Jing, ZHANG Yong, LU Jun-hui, MA Xiang "Multivariate Time Series Modeling Research for Blast Furnace Hot Iron Temperature," Journal of Iron and Steel Research, vol.26, April 2014.

[5] Arif, Fahmi, Suryana, Nanna, Hussin, Burairah, "Cascade Quality Prediction Method Using Multiple PCA+ID3 for Multi-Stage Manufacturing System,” IERI Procedia, vol.4, pp.201-207, 2013.

[6] Ali Azadeh, Mohamad Sadegh Sangari, Alireza Shamekhi Amiri, "A particle swarm algorithm for inspection optimization in serial multi-stage processes", Applied Mathematical Modelling, vol.36, pp.1455-1464, 2012.

[7] Andreas Christmann, Ingo Steinwart, "Support Vector Machines," Information Science and Statistics, 2008.

[8] Xiaolin Huang, Lei Shi, Johan A.K, "Suykens. Asymmetric least squares support vector machine classifiers," Computational Statistics \& Data Analysis, vol.70, pp. 395-405, February 2014.

[9] Huanhuan Yu, Rongda Chen, Guoping Zhang, "A SVM Stock Selection Model within PCA," Procedia Computer Science, vol.31, pp. 406-412, 2014.

[10] Dun Liu, Tianrui Li, Junbo Zhang, "A rough set-based incremental approach for learning knowledge in dynamic incomplete information systems," International Journal of Approximate Reasoning, vol.55, pp. 1764-1786, November 2014.

[11] Mohamad Hosein Refan, Adel Dameshghi, Mehrnoosh Kamarzarrin, "Improving RTDGPS accuracy using hybrid PSOSVM prediction model," Aerospace Science and Technology , vol.37, pp.55-69, 2014. 